\title{
Herpes simplex virus type- I(HSV-I) oncolytic and highly fusogenic mutants carrying the NVI 020 genomic deletion effectively inhibit primary and metastatic tumors in mice
}

\author{
Anna Israyelyan ${ }^{1,2}$, Vladimir N Chouljenko ${ }^{1,2}$, Abolghasem Baghian ${ }^{1,2}$, \\ Andrew T David ${ }^{1,2}$, Michael T Kearney ${ }^{2}$ and Konstantin G Kousoulas*1,2
}

\begin{abstract}
Address: ${ }^{1}$ Division of Biotechnology and Molecular Medicine and Department of Pathobiological Sciences, School of Veterinary Medicine, Louisiana State University, Baton Rouge, LA 70803, USA and 2Department of Pathobiological Sciences, School of Veterinary Medicine, Louisiana State University, Baton Rouge, LA 70803, USA

Email: Anna Israyelyan - aisray1@lsu.edu; Vladimir N Chouljenko - vladimir@vetmed.lsu.edu; Abolghasem Baghian - abaghian@vetmed.lsu.edu; Andrew T David - adavid@vetmed.lsu.edu; Michael T Kearney - mtk@vetmed.lsu.edu; Konstantin G Kousoulas* - vtgusk@lsu.edu

* Corresponding author
\end{abstract}

Published: 2 June 2008

Virology Journal 2008, 5:68 doi:10.1186/1743-422X-5-68

This article is available from: http://www.virologyj.com/content/5/l/68

(C) 2008 Israyelyan et al; licensee BioMed Central Ltd.

This is an Open Access article distributed under the terms of the Creative Commons Attribution License (http://creativecommons.org/licenses/by/2.0), which permits unrestricted use, distribution, and reproduction in any medium, provided the original work is properly cited.
Received: 10 April 2008

Accepted: 2 June 2008

\begin{abstract}
Background: The NVI020 oncolytic herpes simplex virus type-I has shown significant promise for the treatment of many different types of tumors in experimental animal models and human trials. Previously, we described the construction and use of the NVI020-like virus OncSyn to treat human breast tumors implanted in nude mice. The syncytial mutation gKsyn I (Ala-to-Val at position 40) was introduced into the OncSyn viral genome cloned into a bacterial artificial chromosome using double-red mutagenesis in $E$. coli to produce the OncdSyn virus carrying syncytial mutations in both $\mathrm{gB}(\mathrm{syn} 3)$ and $\mathrm{gK}(\operatorname{syn} \mathrm{l})$.
\end{abstract}

Results: The OncdSyn virus caused extensive virus-induced cell fusion in cell culture. The oncolytic potential of the OncSyn and OncdSyn viruses was tested in the highly metastatic syngeneic mouse model system, which utilizes 4TI murine mammary cancer cells implanted within the interscapular region of Balb/c mice. Mice were given three consecutive intratumor injections of OncSyn, OncdSyn, or phosphate buffered saline four days apart. Both OncSyn and OncdSyn virus injections resulted in significant reduction of tumor sizes $(p<0.05)$ compared to control tumors. Virus treated mice but not controls showed a marked reduction of metastatic foci in lungs and internal organs. Mouse weights were not significantly impacted by any treatment during the course of the entire study $(p=0.296)$.

Conclusion: These results show that the attenuated, but highly fusogenic OncSyn and OncdSyn viruses can effectively reduce primary and metastatic breast tumors in immuncompetent mice. The available bac-cloned OncSyn and OncdSyn viral genomes can be rapidly modified to express a number of different anti-tumor and immunomodulatory genes that can further enhance their antitumor potency. 


\section{Background}

Recent advances in molecular virology have enabled investigators to construct viruses that selectively destroy cancer cells (oncolytic virotherapy). Genetically engineered viruses belonging to different viral families have been evaluated for their potential as therapeutic agents in the treatment of malignant tumors [1-4]. Efficient replication, cell lysis and spread of HSV, and their natural broad host range make them attractive candidates as oncolytic viral agents [5-7]. Furthermore, the recent availability of cloned HSV genomes into bacterial artificial chromosome vectors greatly facilitates the rapid construction of new recombinant viruses carrying multiple transgenes of interest [8-10]. Tumor treatment with oncolytic HSV has been shown to induce anti-tumor immune responses [11-15]. Although the majority of people are seropositive for HSV1 , oncolytic virotherapy with HSV is not limited by preexisting anti-HSV immunity $[16,17]$, and in at least one example, preexisting immunity to HSV-1 enhanced antitumor immune responses [18].

Recently, the NV1020 oncolytic herpes simplex virus type1(HSV-1) was shown to have significant promise for the treatment of many different types of tumors in preclinical studies in experimental animals as well as in human clinical trials [17,19-22]. The main advantage of this virus over other HSV oncolytic viruses is that it expresses one of the two original $\gamma_{1} 34.5$ genes allowing the virus to replicate more efficiently, while safety is not compromised [23-26]. The $\gamma_{1} 34.5$ gene is a major neurovirulence gene and an inhibitor of cellular apoptosis. Deletion of this gene drastically attenuates the virus and restricts viral growth to cancer cells because of their lack of intact apoptotic mechanisms $[27,28]$. Preclinical studies in mice as well as phase I/II human trials have revealed that oncolytic HSV-1 viruses having both $\gamma_{1} 34.5$ genes deleted did not spread efficiently within tumors [29]. In contrast, deletion of one of the two $\gamma_{1} 34.5$ genes drastically attenuated the virus, while allowing improved virus replication and spread in tumor cells [23-25]. The NV1020 was originally constructed for vaccine purposes and it contains HSV-2 viral sequences coding for glycoproteins gD, gG, gI and $\mathrm{gE}$ to facilitate production of anti-HSV-2 immune responses [24].

HSV can be transmitted from cell-to-cell by causing limited amounts of virus-induced cell fusion, thus avoiding the extracellular environment. Specific mutations within viral glycoproteins are known to greatly enhance virusinduced cell fusion. Specifically, syncytial mutations that cause extensive virus-induced cell fusion can arise in at least two of the glycoprotein genes: the UL27 gene, encoding glycoprotein $\mathrm{B}(\mathrm{gB})[30-32]$, and the UL53 gene, coding for glycoprotein $\mathrm{K}(\mathrm{gK})$ [33,34]. Work in our laboratory has shown that $\mathrm{gK}$ functions as a heterodimer with the UL20 viral protein and the UL20/gK heterodimer is necessary for virus-induced cell fusion $[35,36]$.

The HSV-1 oncolytic virus Onc was constructed based on the NV1020 genomic arrangement with the exception that there are no genomic re-arrangements and no HSV-2 genes inserted within the viral genome. Recently, we reported that the OncSyn virus carrying a syncytial mutation in $\mathrm{gB}$, enabling the virus to spread among cells by virus-induced cell fusion, replicated efficiently in breast cancer cells in vitro and drastically reduced tumor volumes in vivo [37]. In this study we constructed and tested the OncdSyn virus, which in addition to the gBsyn 3 mutation also carried the gKsyn 1 mutation known to enable the virus to fuse even difficult to fuse cells [38]. Intra-tumor injections of either virus effectively reduced tumor volumes as well as inhibited tumor metastases to internal organs.

\section{Results \\ Construction and characterization of the Oncolytic HSV-I mutant virus OncdSyn}

Previously, we described the construction and use of the NV1020-like virus OncSyn to treat human breast cancer utilizing a nude mouse xenograft model [37]. To further increase the ability of the OncSyn virus to cause virusinduced cell fusion, the syncytial mutation gKsyn1 (Alato-Val at position 40) known to cause virus-induced cell fusion of even hard to fuse cells [38] was introduced into the OncSyn viral genome cloned into a bacterial artificial chromosome (bac) using the markerless double-red mutagenesis method [39]. The resultant OncdSyn virus carried syncytial mutations in both $\mathrm{gB}$ (syn3) and $\mathrm{gK}$ (syn1) (Fig. 1). As we reported previously for the OncSyn virus, the bac-cloned OncdSyn viral genome was subjected to PCR-diagnostic analysis and direct sequencing of specific genomic loci to confirm the presence of the syn 3 and syn 1 mutations and the previously engineered deletion/insertion at the $\gamma_{1} 34.5$ locus (not shown, Materials and Methods).

\section{Phenotypic characteristics of the OncSyn and OncdSyn viruses on Vero and 4TI cells}

The plaque morphology of the HSV-1(F), OncSyn and OncdSyn viruses was examined on Vero cells and 4T1 cancer cells (Balb/c spontaneous mammary adenocarcinomaderived) [40] as described in Materials and Methods (Fig. 2 ). Plaque morphologies were visualized on Vero and 4T1cells at 48 hours post infection (hpi) by immunohistochemistry using a polyclonal anti-HSV-1 antibody (Fig. 2a-f). Mouse cells are known to be resistant to HSV-1 infection $[41,42]$. Consequently, viral plaques generated by all three viruses tested were substantially smaller on 4T1 mouse cancer cells (Fig. 2d, e, f) in comparison to Vero cells (Fig. 2a, b, c). Specifically, the HSV-1(F) wild- 




Figure I

Schematic representation of the genomic structures of the oncolytic recombinant viruses OncSyn and OncdSyn. (a) Representation of the prototypic arrangement of the HSV-I genome with the unique long (UL) and unique short (US) regions flanked by the terminal repeat (TR) and internal repeat (IR) regions. (b) Approximate locations of the gB and gK genes. (c) An expansion of the inverted repeat region showing the approximate locations of UL54, UL55, UL56, $\alpha$, $\gamma_{1} 34.5, \alpha 4, \alpha 22$ and US2 genes. (d) Schematic of the DNA fragment cloned into plasmid pJM-R, which was used for insertion of the HcRed gene cassette into the viral genome in place of the NVI020 genomic deletion as described in Materials and Methods.

type virus, which does not cause extensive virus-induced cell fusion, produced viral plaques on $4 \mathrm{~T} 1$ cells that were approximately 10 -fold smaller than those produced on Vero cells (Fig. 2d and 2a). In contrast, the OncSyn and OncdSyn viruses produced syncytial plaques on both cell lines tested (Fig. 2b, c, e, f); however, both the OncSyn and OncdSyn viral plaques on 4T1 cells were larger than those produced by the HSV-1(F) wild-type virus (Fig. $2 e$ and $2 \mathrm{f}$ compared to $\mathrm{d}$ ). The OncdSyn virus appeared to cause more pronounced virus-induced cell fusion on both Vero and 4T1 cells (Fig. 2c and 2f). In addition, the OncdSyn viral plaques emitted strong red fluorescence due to constitutive expression of the red fluorescence protein (RFP) expressed under the elongation factor $1 \alpha$ (EF$1 \alpha$ ) promoter control (Fig. $2 \mathrm{~g}$ and $2 \mathrm{~h}$ ), as it was previously reported for the OncSyn virus [37].

\section{Kinetics of viral replication on Vero and 4TI cells}

HSV-1(F) and OncSyn viruses replicated to similar titers in Vero cells, while the OncdSyn virus consistently replicated to titers that were a half-log lower than either HSV$1(\mathrm{~F})$, or OncSyn viruses. The kinetics of viral replication were substantially slower in 4T1 cells than in Vero cells, and final titers in $4 \mathrm{~T} 1$ cells were more than two logs lower for HSV-1(F) and OncSyn, while OncdSyn viral titers were more than three logs lower on 4T1 cells than in Vero cells. In addition, OncdSyn viral titers were approximately one log lower than the HSV-1(F) and OncSyn viral titers on 4T1 cells (Fig. 3).

\section{Intra-tumor virotherapy}

$4 \mathrm{~T} 1$ cells were injected subcutaneously in the interscapular regions of Balb/c female mice. When the palpable tumors reached the volume of approximately 80-90 $\mathrm{mm}^{3}$, mice were injected with three consecutive intratumor injections of OncSyn and OncdSyn viruses or PBS (control) every four days as described in Materials and Methods. At the onset of viral intratumor injections, tumor sizes appeared similar in size for all three groups of mice ( $p>0.05$ ). Intratumor treatment with either OncSyn or OncdSyn virus caused a substantial reduction of tumor volumes in comparison to the PBS-treated control group of mice $(\mathrm{p}<0.05)$. There was no significant difference in the reduction of tumor sizes in the two viral groups when compared to each other ( $\mathrm{p}>0.05$ ) (Fig. 4a). Analysis of mouse weights during the course of the study did not show significant differences among the three groups, thus the efficacy of treatments was not affected by differential 




HSV-1(F)



OncSyn

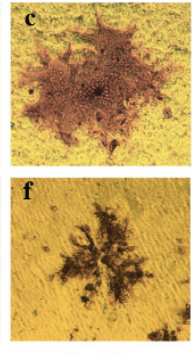

OncdSyn



OncdSyn
Figure 2

Plaque morphology of the HSV-I (F), OncSyn and OncdSyn viruses. Nearly confluent Vero (a-c) and 4TI (df) cell monolayers were infected with wild-type HSV-I (F) (a, d), OncSyn (b, e) and OncdSyn (c, f) viruses. Individual viral plaques were visualized $48 \mathrm{hr}$ post infection by immunohistochemistry and photographed with a phase contrast microscope. Vero $(\mathrm{g})$ and 4TI $(\mathrm{h})$ cells were infected with OncdSyn virus. Viral plaques were photographed $48 \mathrm{hr}$ postinfection with a fluorescent microscope.

weight gain/loss in the groups (not shown) ( $\mathrm{p}=0.296)$. Representative tumors were excised immediately after mice were sacrificed. Typically, tumors treated with the PBS control injections appeared substantially larger than those treated with either the OncSyn or OncdSyn viruses (Fig. 4b).

The metastatic potential of the primary $4 \mathrm{~T} 1$ tumor to internal organs after oncolytic or control therapy was assessed by gross and microscopic pathological examination of internal organs. In the first experimental protocol described above, mouse tumors were allowed to grow to approximately $80-90 \mathrm{~mm}^{3}$ and mice were sacrificed at 42 days post tumor cell implantation. In this experiment, mouse lungs from all three groups of mice (PBS, OncSyn, OncdSyn) had numerous metastatic foci, which were too numerous to be accurately counted (not shown). However, tumor foci in liver and spleen were substantially reduced in OncSyn and OncdSyn-treated mice in comparison to PBS-treated control mice (Table 1, Fig. 5). Specifically, all mice in the PBS group had metastatic nodes in liver, spleen, or kidneys. Some of the mice had tumors in all three organs (Fig. 5). Importantly, there were no metastatic tumors observed in the kidneys of virus-treated mice (Table 1).

To better assess the potential of oncolytic virotherapy to reduce metastatic tumors in internal organs, a second experiment was performed in a similar fashion to the previous one with the exception that in the new experiment tumors were allowed to grow to approximately 35-40 $\mathrm{mm}^{3}$ in volume and mice were sacrificed at day 33 post tumor cell implantation after treatment with either a

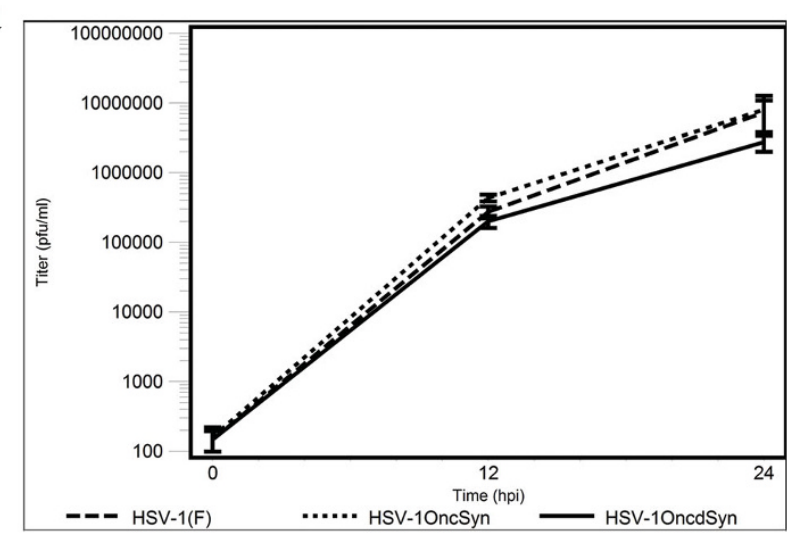

b

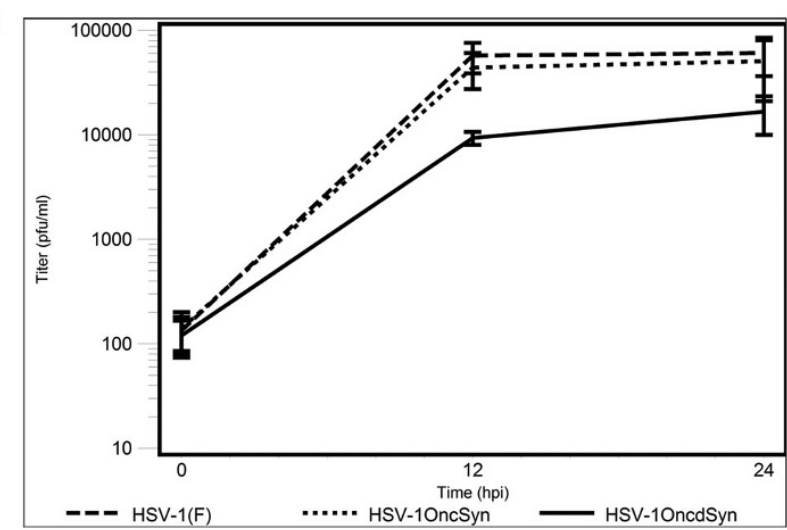

Figure 3

Comparative kinetics of viral replication of wild-type HSV-I(F) and mutant viruses OncSyn and OncdSyn grown on Vero and 4TI cells. Near confluent monolayers of Vero (a) and 4TI (b) cells were infected at an MOI of 2 with each virus, incubated at $37^{\circ} \mathrm{C}$ and the numbers of infectious virions were determined at different times post infection. Viral titers (mean pfu at each time point) are shown in logarithmic scale. The error bars represent means \pm 2 standard errors.

OncdSyn or PBS. Lungs of OncdSyn-treated mice appeared to be practically devoid of metastatic tumors with only two mice having two nodes each. In contrast, all PBS-treated mice had multiple metastatic tumors in their lungs (Table 2, Fig. 6a and 6b). These results were confirmed by pathological examination of paraffin-embedded lung sections stained with Hematoxylin \& Eosin (H\&E) staining, which revealed the absence of tumors in OncdSyn samples, while PBS-treated control samples had numerous visible tumor foci (Fig. 6c-f).

\section{Discussion}

The oncolytic HSV-1-based virus NV1020 has shown strong promise for treatment of different tumors in animal models and human clinical trials [17,19-22]. To facil- 




b

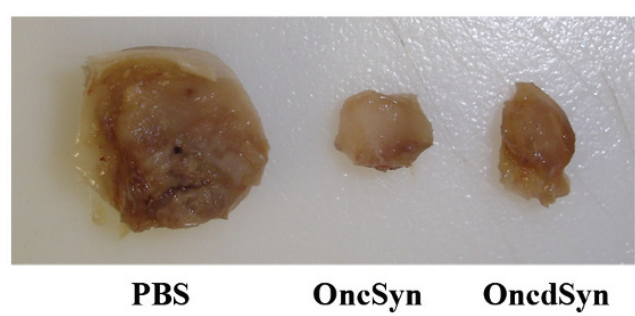

Figure 4

Intra-tumor treatment with OncSyn and OncdSyn viruses. (a) Balb/c mice were implanted subcutaneously in the interscapular area with I $\times 10^{5}$ viable $4 \mathrm{TI}$ cells. Tumors were measured using a digital caliper at defined time intervals prior and after treatment ( $x$ axis). Tumors were injected with either OncSyn, OncdSyn viruses, or PBS when tumors reached approximately $80-90 \mathrm{~mm}^{3}$ in volume. Tumor volumes were measured prior to (negative values on the $x$ axis) and after the injections. " 0 " on $X$ axis represents the day of the first injection. The tumor volumes were determined from the formula: volume $=($ length $\times$ width $\times$ height $) / 2$. Arrows indicate the days when therapy was administered. The error bars represent means \pm 2 standard errors. (b) Tumors were excised at 42 days post implantation and visually examined. Panel shows representative tumors from virus and PBS treated animals.

itate the construction of recombinant viruses carrying multiple transgenes of interest, we cloned the NV1020like HSV-1 recombinant virus OncSyn into a bacterial artificial chromosome (bac) vector. The OncSyn virus specifies a syncytial mutation in gB (Arg-to-His change at aa 858 ) that increases its ability to spread in tumor cells via virus-induced cell fusion [37]. In this study, we introduced the syncytial mutation syn1 (Ala-to-Val change at aa 40) within the $\mathrm{gK}$ gene to further enhance the fusogenicity of the new virus OncdSyn. The OncdSyn virus reduced primary tumor sizes and inhibited metastases to distal organs in the 4T1 syngeneic mouse model system.



Liver lobe

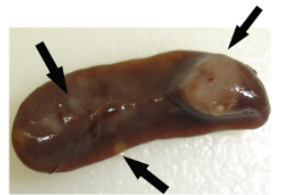

Spleen

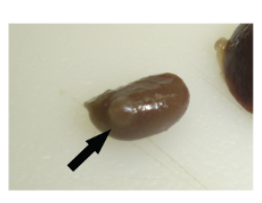

Kidney
Figure 5

Gross pathological examination of metastatic tumor nodules on internal organs. Liver lobe, spleen, and kidney from a PBS-treated mouse carrying metastatic tumors (arrows) evaluated by gross pathological examination. Panel shows internal organs derived from a representative PBStreated mouse.

The syn 1 mutation within $\mathrm{gK}$ has been shown to produce extensive virus-induced cell fusion in all cells tested. In comparison, the gBsyn3 mutation produced virusinduced cell fusion in most cells, but it was unable to fuse certain hard to fuse cells, such as Hep-2 cells derived from human laryngeal carcinoma [38]. Therefore, to further increase the ability of the OncSyn virus to fuse all types of cells, we generated the OncdSyn virus carrying both the gBsyn 3 and gKsyn 1 mutations. As expected, the OncdSyn virus caused extensive virus-induced cell fusion and fused Hep-2 cells, while the OncSyn virus did not (not shown). Furthermore, the OncdSyn virus caused more extensive fusion than OncSyn in both Vero and 4T1 cells. The OncdSyn virus appeared to produce intact syncytia that remained attached to the cell culture flasks, while the OncSyn virus-induced syncytia contained infected single cells, which detached easier than the OncdSyn-infected syncytia. This phenomenon has been previously observed for the $\mathrm{gB}$ and $\mathrm{gK}$ syncytial mutations and it is probably due, in part, to the extensive virus-induced cell fusion caused by the gK syncytial mutation, which appears to also fuse internal membranes such as nuclear membranes in addition to plasma membranes of cells (Kousoulas, unpublished). Viral titers of the OncdSyn virus were lower in Vero cells than titers of the OncSyn virus and substantially lower than titers of the OncSyn virus in 4T1 cells. Typically, HSV-1 syncytial mutants produce lower viral titers than their parental wild-type viruses, most likely because of their direct effect on cellular membranes. In this regard, the increased ability of the OncdSyn virus to cause extensive virus-induced cell fusion is probably responsible for the observed decrease in viral titers in comparison to the OncSyn virus.

Defects of viral replication and spread in mouse cancer cells have been described in the literature for oncolytic herpesviruses $[11,14]$. HSV-1 does not replicate efficiently in mouse cell lines $[41,42]$ most likely because it cannot as efficiently utilize the mouse nectin-1 receptor, which is approximately $5 \%$ different in its amino acid sequence to 
Table I: Metastatic nodes in internal organs

\begin{tabular}{|c|c|c|c|c|c|}
\hline $\begin{array}{l}\text { Experimental } \\
\text { groups }\end{array}$ & $\begin{array}{l}\text { No. of mice in } \\
\text { group }\end{array}$ & $\begin{array}{l}\text { No. of mice with } \\
\text { metastases in } \\
\text { internal organs }\end{array}$ & $\begin{array}{l}\text { No. of mice with } \\
\text { metastases in liver }\end{array}$ & $\begin{array}{l}\text { No. of mice with } \\
\text { metastases in } \\
\text { spleen }\end{array}$ & $\begin{array}{l}\text { No. of mice with } \\
\text { metastases in } \\
\text { kidney }\end{array}$ \\
\hline PBS & 9 & 9 & 6 & 7 & 3 \\
\hline OncSyn & 7 & 4 & 2 & 2 & 0 \\
\hline OncdSyn & 7 & 3 & 1 & 2 & 0 \\
\hline
\end{tabular}

Experimental animals were sacrificed on day 42 post-injection of $4 \mathrm{TI}$ cells and the internal organs were removed and examined for metastases formation by gross pathological evaluation as described in Materials and Methods.

the human nectin-1 receptor [43]. Nectin-1 is also known to facilitate virus-induced cell fusion and virus-spread [44]. Consequently, both OncSyn and OncdSyn viruses replicated much less efficiently in 4T1 cells than in Vero cells. In this regard, the limited replication and spread of these viruses in $4 \mathrm{~T} 1$ cells would be expected to adversely affect their oncolytic ability in 4T1-derived tumors in vivo. Previously, we reported that the OncSyn virus effectively reduced primary human breast cancer tumors in nude mice [37]. The disadvantage of the MDA-MB-435S human breast tumors is that these tumors would be rapidly eliminated if they were implanted in immunocompetent mice. Therefore, we chose the $4 \mathrm{~T} 1 / \mathrm{Balb} / \mathrm{c}$ mouse model system for additional testing of both the previously constructed OncSyn virus as well as the newly constructed OncdSyn virus. Both OncSyn and OncdSyn viruses substantially reduced the growth of $4 \mathrm{~T} 1$ tumors compared to the PBS controls, despite the fact that these viruses did not efficiently replicate in 4T1 cells in cell culture. Apparently, viral replication and infectious virus production in cell cultures did not correlate with the oncolytic efficacy of these viruses, because the OncdSyn virus reduced tumor volumes equally-well with the OncSyn virus, despite the fact that OncdSyn replicated approximately less than half a log than the OncSyn virus in 4T1 cells. Therefore, the relative increased ability of the OncdSyn virus to destroy tumors in vivo must be attributed to its enhanced fusogenicity.

Multiple murine tumor models have been used as preclinical settings for therapeutic purposes. The 4T1 mammary carcinoma model has several distinct advantages to be used as such model. It is regarded as a highly physiological, clinically-relevant mouse model that closely resembles stage IV human breast cancer in its properties [40]. $4 \mathrm{~T} 1$ cells are considered to be very weakly immunogenic (relative antigenic strength is less than 0.01 with 9.9 being the most immunogenic) $[45,46]$, and they spontaneously metastasize to distal parts of the body [40,47]. Metastatic tumor foci in liver and spleen were substantially reduced in OncSyn and OncdSyn-treated mice in comparison to PBS-treated control mice. Reduction of metastatic foci in internal organs (lung, spleen, kidney and liver) was dependent on the size of the original 4T1 tumor, as well as the time of necropsy post implantation of tumor cells. Specifically, there was drastic reduction in tumor foci detected in lungs when the initial tumor size treated with the virus was approximately $35-40 \mathrm{~mm}^{3}$ and necropsies were performed at 33 days after tumor implantation. Furthermore, lungs appeared to have the same number of metastatic foci with PBS-treated controls when the initial treated tumors where $80-90 \mathrm{~mm}^{3}$ and necropsies were performed at day 42 after tumor implantation. This metastatic pattern revealed that lungs were the primary metastatic site of the subcutaneous implanted 4T1 cells. Regardless of the size of the initial tumor treated and the time of necropsies post tumor implantation, it was evident that OncSyn and OncdSyn viruses appeared to efficiently reduce the growth of the primary tumor as well as substantially inhibit or eliminate formation of metastatic foci.

It is highly likely that reduction of the primary tumor after oncolytic virotherapy with the OncSyn and OncdSyn viruses is responsible for the observed reduction in the formation of secondary tumor foci, since treatment of the smaller (35-40 $\mathrm{mm}^{3}$ ) tumors appeared to drastically reduce lung metastases. Alternatively, it is possible that anti-tumor immune responses were elicited by exposure of tumor antigens after destruction of 4T1 cells within the primary tumor by the OncSyn and OncdSyn viruses. In this regard, a fusogenic oncolytic HSV-1 Synco-2D was reported to elicit anti-tumor immune responses when studied in a similar animal model of mammary carcinoma utilizing 4T1 cells [14]. A strong T-cell response was reported also by an HSV-2 derivative oncolytic virus FusOn-H2 effectively treating primary and metastatic mammary tumors in vivo [15].

\section{Conclusion}

Overall, our results showed that both OncSyn and OncdSyn viruses can efficiently reduce the primary and metastatic growth of $4 \mathrm{~T} 1$ tumors established in immunocompetent mice. It is expected that these viruses would be even more efficacious against human breast cancer tumors by virtue of the fact that they can replicate substantially more efficiently (more than one log) in human than mouse cells. The availability of both OncSyn and 



PBS



OncdSyn

Figure 6

Therapeutic effect of OncdSyn virus on lung metastases. (a, b) Gross appearance of excised lungs of representative mice from PBS control and OncdSyn treated groups. (cf) Lung tissues were stained with H\&E and examined. Representative stained sections are shown for PBS (c, d) and OncdSyn (e, f) groups at $40 \times(c, e)$ and $100 \times(d, f)$ magnifications. Metastatic foci are represented by arrows (c, d).

OncdSyn viruses as bacterial artificial chromosomes will enable the generation of additional recombinant viruses that carry multiple anti-tumor and immunomodulatory transgenes, which could further enhance the anti-tumor efficacy of these viruses.

\section{Materials and methods \\ Cells}

African green monkey kidney (Vero) cells and mouse mammary tumor cells (4T1) [40] were obtained from the American Type Culture Collection (Manassas, VA). Vero cells were maintained in Dulbecco's modified Eagle's medium (Gibco-BRL; Grand Island, N.Y.), supplemented with $10 \%$ fetal calf serum (FCS) and antibiotics. 4T1 cells were maintained in RPMI 1640 medium (Hyclone, Logan, UT) containing 10\% FCS. The cultures were main-

tained at $37^{\circ} \mathrm{C}$ in a humidified atmosphere of $5 \% \mathrm{CO}_{2} /$ $95 \%$ air.

\section{Construction of the doubly fusogenic recombinant virus HSV-I OncdSyn}

The previously published OncSyn viral genome recovered as a bacterial artificial chromosome (bac) into E. coli (pOncSyn) [37] was used for the construction of pOncdSyn bac plasmid utilizing a new methodology - the double-red mutagenesis technique in E. coli [39] enabling the markerless introduction of the gKsyn 1 mutation (AlatoVal at aa 40). The OncdSyn virus was recovered after transfection of Vero cells with the pOncdSyn plasmid. The OncdSyn viral genome and the pOncdSyn bac were extensively characterized by diagnostic PCR and DNA sequencing to ensure the stability of the viral genomes, the presence of the parental Onc deletions and the presence of the gKsyn 1 mutation within the gK gene, as described previously for the OncSyn virus [37].

\section{Phenotypic characterization and replication kinetics of the OncSyn and OncdSyn viruses}

Cells (both Vero and 4T1) were seeded into 6-well plates and infected the following day (when they reached approximately 95\% confluency) with the OncSyn or OncdSyn viruses at a multiplicity of infection (MOI) ranging from 0.001-1 plaque forming units per cell (PFU/ cell). Cells were cultured in a maintenance medium (containing 2\% FCS) and were left for 2 days to allow for the plaques and the cell fusion to develop. Photographs of the infected cells were taken using a fluorescence microscope. For assessment of viral plaque morphologies, Vero and 4T1 cells were infected with HSV-1(F), OncSyn or OncdSyn viruses and visualized after immunohistochemistry at 48 hours post-infection (h.p.i.) using horseradish peroxidase-conjugated anti-HSV antibody (Dako, Carpinteri, CA) and Novared substrate development kit (VectorLabs, Burlingame, CA).

To determine the replication kinetics of the viruses, onestep growth kinetics were performed as described previously $[48,49]$. Briefly, nearly confluent monolayers of either Vero or $4 \mathrm{~T} 1$ cells were infected with each virus at an MOI of 2 at $4^{\circ} \mathrm{C}$ for $1 \mathrm{~h}$. Thereafter, virus was allowed to penetrate for $2 \mathrm{~h}$ at $37^{\circ} \mathrm{C}$. Any remaining extracellular virus was inactivated by low-pH treatment with phosphate buffered saline at $\mathrm{pH}$ 3.0. Cells and supernatants were harvested immediately thereafter $(0 \mathrm{~h})$ or after 12 or $24 \mathrm{~h}$ of incubation at $37^{\circ} \mathrm{C}$. Virus titers were determined by endpoint titration of virus stocks on Vero cells.

\section{Animal experiments}

Female Balb/c mice were obtained from Harlan (Indianapolis, IN) and housed in an animal room which was kept at $25^{\circ} \mathrm{C}$ with a 12 hour light-dark cycle. All experimental 
Table 2: Metastatic nodes in lungs

\begin{tabular}{|c|c|c|c|c|c|c|c|c|c|}
\hline \multirow[t]{2}{*}{ Experimental groups } & \multirow[t]{2}{*}{ No. of mice in group } & \multicolumn{8}{|c|}{ No. of metastatic nodes in lungs of experimental animals ${ }^{a}$} \\
\hline & & mousel & mouse2 & mouse3 & mouse4 & mouse5 & mouse6 & mouse7 & mouse8 \\
\hline PBS & 7 & 3 & 5 & 3 & 18 & 3 & 10 & I & \\
\hline OncdSyn & 8 & 2 & 0 & 0 & 0 & 2 & 0 & 0 & 0 \\
\hline
\end{tabular}

Experimental animals in PBS and OncdSyn groups were sacrificed on day 33 post-inoculation of 4TI cells and the lungs were removed and examined for metastatic node formation as described in Materials and Methods. aPBS > OncdSyn (Wilcoxon Two-Sample test, $p=0.002$ ).

procedures involving animals were approved by the institutional animal care and use committee (IACUC) of the Louisiana State University. At 6-7 weeks of age the animals (19-20 g body weight) were implanted subcutaneously in the interscapular area with $1 \times 10^{5}$ viable $4 \mathrm{~T} 1$ cells suspended in $0.2 \mathrm{ml}$ of PBS using a 27 gauge needle. Body weights were determined weekly, and tumor sizes were monitored beginning 7 days after tumor inoculation by direct measuring with a digital microcaliper. Tumor volumes were calculated using the following formula: volume $=($ length $\times$ width $\times$ height $) / 2$. At an average tumor volume of approximately $80-90 \mathrm{~mm}^{3}$ (first experiment) or $35-40 \mathrm{~mm}^{3}$ (second experiment), animals were randomized into 3 groups (first experiment) or 2 groups (second experiment) using a randomization plan. The groups of mice received 3 intratumoral injections of the OncSyn, OncdSyn viral particles, or PBS every four days for the first experiment and injections of the OncdSyn or PBS every third day for the second experiment. Each tumor was injected with approximately $1 \times 10^{6}$ viruses per injection in $250 \mu \mathrm{l}$ volume, while control mice received $250 \mu \mathrm{l}$ of PBS. Injections were performed slowly at 3 different sites per tumor. On day 42 for the first experiment and day 33 for the second experiment after initial tumor cell implantation, mice were humanely euthanized in a $\mathrm{CO}_{2}$ chamber and subjected to gross as well as microscopic histological examination. Lung and other internal organ metastases were counted using a dissecting microscope after placing the resected organs in fixative for 24 hours. The primary tumor site, lungs, heart, liver, spleen, and kidneys from each animal were fixed in $10 \%$ neutral buffered formalin, trimmed, paraffin embedded, sectioned, stained with hematoxylin and eosin (H\&E), and evaluated by light microscopy.

\section{Statistical methods and analyses}

The SAS $^{\circledast}$ statistical package (Version 9.1.3) was used for the analyses of the in vivo studies. Distributions were examined for normality using the UNIVARIATE procedure with a Shapiro-Wilk test of normality. For the repeated measures part of the analyses of tumor volumes and tumor weights, the GLM procedure was used to conduct a repeated measures design analyzed as a split-plot arrangement of treatments with TREATMENT (OncSyn,
OncdSyn, and PBS) and MOUSE within TREATMENT as main plot factors. Subplot factors included PERIOD (days of measurements) and TREATMENT by PERIOD interaction. When overall analyses determined significance $(p=$ $0.05)$, Tukey's HSD test was used to examine pairwise differences for main effects, and pairwise comparisons of least square means with regard to interaction effects were examined with preplanned t-tests. The Wilcoxon TwoSample test was used to analyze the difference of lung metastatic node counts between PBS and OncdSyn groups.

\section{Competing interests}

The authors declare that they have no competing interests.

\section{Authors' contributions}

AI performed most of the experiments and participated in drafting the manuscript, VNC participated in the construction and characterization of the viruses, $\mathrm{AB}$ was involved in the design and conduction of in vivo studies, ATD participated in pathological analysis and interpretation of data, MTK performed the statistical analyses, KGK was overall responsible for the project and for the preparation of the manuscript.

\section{Acknowledgements}

This work was supported by the grant "Novel Cancer Treatment Modalities" from the Louisiana Governor's Biotechnology Initiative (GBI), Louisiana Board of Regents and by the grant R0I Al43000 from NIH:NIAID to K.G.K. The project was also supported by the Louisiana Gene Therapy Program of the LSU Health Sciences Center, New Orleans. The authors gratefully acknowledge BioMMED's support by the LSU School of Veterinary Medicine and helpful discussions with Marlene Orandle and Karen Peterson (Department of Pathobiological Sciences, School of Veterinary Medicine, Louisiana State University).

\section{References}

I. Chiocca EA: Oncolytic viruses. Nat Rev Cancer 2002, 2:938-950.

2. Kirn D: Oncolytic virotherapy as a novel treatment platform for cancer. Ernst Schering Res Found Workshop 2003:89-I05.

3. Nemunaitis J, Edelman J: Selectively replicating viral vectors. Cancer Gene Ther 2002, 9:987-1000.

4. Nemunaitis J: Live viruses in cancer treatment. Oncology (Williston Park) 2002, I 6: |483-|492. discussion |495-I487

5. Lachmann R: Herpes simplex virus-based vectors. Int J Exp Pathol 2004, 85: I77-190.

6. Fu X, Nakamori M, Tao L, Amato R, Zhang X: Antitumor effects of two newly constructed oncolytic herpes simplex viruses against renal cell carcinoma. Int J Oncol 2007, 30: I56 I-I567. 
7. Fu X, Tao L, Cai R, Prigge J, Zhang X: A mutant type 2 herpes simplex virus deleted for the protein kinase domain of the ICP IO gene is a potent oncolytic virus. Mol Ther 2006, 13:882-890.

8. Meseda CA, Schmeisser F, Pedersen R, Woerner A, Weir JP: DNA immunization with a herpes simplex virus 2 bacterial artificial chromosome. Virology 2004, 3 I 8:420-428.

9. Schmeisser F, Weir JP: Incorporation of a lambda phage recombination system and EGFP detection to simplify mutagenesis of Herpes simplex virus bacterial artificial chromosomes. BMC Biotechnol 2007, 7:22.

10. Jeyaretna DS, Kuroda T: Recent advances in the development of oncolytic HSV-I vectors: 'arming' of HSV-I vectors and application of bacterial artificial chromosome technology for their construction. Curr Opin Mol Ther 2007, 9:447-466.

1I. Thomas DL, Fraser NW: HSV-I therapy of primary tumors reduces the number of metastases in an immune-competent model of metastatic breast cancer. Mol Ther 2003, 8:543-55I.

12. Todo T, Rabkin SD, Sundaresan P, Wu A, Meehan KR, Herscowitz $H B$, Martuza RL: Systemic antitumor immunity in experimental brain tumor therapy using a multimutated, replicationcompetent herpes simplex virus. Hum Gene Ther 1999 , I 0:274 I-2755

13. Miller CG, Fraser NW: Requirement of an integrated immune response for successful neuroattenuated HSV-I therapy in an intracranial metastatic melanoma model. Mol Ther 2003 7:74I-747.

14. Nakamori M, Fu X, Rousseau R, Chen SY, Zhang X: Destruction of nonimmunogenic mammary tumor cells by a fusogenic oncolytic herpes simplex virus induces potent antitumor immunity. Mol Ther 2004, 9:658-665.

15. Li H, Dutuor A, Fu X, Zhang X: Induction of strong antitumor immunity by an HSV-2-based oncolytic virus in a murine mammary tumor model. J Gene Med 2007, 9:161-169.

16. Hoffmann D, Bayer W, Wildner O: Local and distant immunemediated control of colon cancer growth with fusogenic membrane glycoproteins in combination with viral oncolysis. Hum Gene Ther 2007, 18:435-450.

17. Kemeny N, Brown K, Covey A, Kim T, Bhargava A, Brody L, Guilfoyle B, Haag NP, Karrasch M, Glasschroeder B, et al:: Phase I, OpenLabel, Dose-Escalating Study of a Genetically Engineered Herpes Simplex Virus, NVI020, in Subjects with Metastatic Colorectal Carcinoma to the Liver. Hum Gene Ther 2006 , 17:1214-1224.

18. Miller CG, Fraser NW: Role of the immune response during neuro-attenuated herpes simplex virus-mediated tumor destruction in a murine intracranial melanoma model. Cancer Res 2000, 60:57|4-5722.

19. Cozzi PJ, Burke PB, Bhargav A, Heston WD, Huryk B, Scardino PT, Fong $Y$ : Oncolytic viral gene therapy for prostate cancer using two attenuated, replication-competent, genetically engineered herpes simplex viruses. Prostate 2002, 53:95-100.

20. Cozzi PJ, Malhotra S, McAuliffe P, Kooby DA, Federoff HJ, Huryk B, Johnson P, Scardino PT, Heston WD, Fong Y: Intravesical oncolytic viral therapy using attenuated, replication-competent herpes simplex viruses G207 and Nv1020 is effective in the treatment of bladder cancer in an orthotopic syngeneic model. Faseb J 200I, 15: I306-1308.

21. Gutermann A, Mayer E, Von Dehn-Rothfelser K, Breidenstein C Weber M, Muench M, Gungor D, Suehnel J, Moebius U, Lechmann M: Efficacy of oncolytic herpesvirus NV 1020 can be enhanced by combination with chemotherapeutics in colon carcinoma cells. Hum Gene Ther 2006, 17:1241-1253.

22. McAuliffe PF, Jarnagin WR, Johnson P, Delman KA, Federoff $H$, Fong $Y$ : Effective treatment of pancreatic tumors with two multimutated herpes simplex oncolytic viruses. J Gastrointest Surg 2000, 4:580-588.

23. Advani SJ, Chung SM, Yan SY, Gillespie GY, Markert JM, Whitley RJ, Roizman B, Weichselbaum RR: Replication-competent, nonneuroinvasive genetically engineered herpes virus is highly effective in the treatment of therapy-resistant experimental human tumors. Cancer Res 1999, 59:2055-2058.

24. Meignier B, Longnecker R, Roizman B: In vivo behavior of genetically engineered herpes simplex viruses $R 7017$ and $R 7020$ construction and evaluation in rodents. J Infect Dis 1988 , | 58:602-6|4.
25. Meignier B, Martin B, Whitley RJ, Roizman B: In vivo behavior of genetically engineered herpes simplex viruses $R 7017$ and R7020. II. Studies in immunocompetent and immunosuppressed owl monkeys (Aotus trivirgatus). J Infect Dis 1990 162:3|3-32|

26. Cadoz M, Seigneurin JM, Mallaret MR, Baccard C, Morand P: Phase I trial of R7020: A live attenuated recombinant herpes simplex virus (HSV) candidate vaccine. In Program and Abstacts of the 32nd Interscience Conference on Antimicrobial Agents and Chemotherapy American Society of Microbiology, Washington, D.C; 1992

27. Chou J, Kern ER, Whitley RJ, Roizman B: Mapping of herpes simplex virus-I neurovirulence to gamma 134.5 , a gene nonessential for growth in culture. Science 1990, 250:1262-1266.

28. Chou J, Roizman B: The gamma I(34.5) gene of herpes simplex virus I precludes neuroblastoma cells from triggering total shutoff of protein synthesis characteristic of programed cell death in neuronal cells. Proc Natl Acad Sci USA 1992, 89:3266-3270.

29. Kramm CM, Chase M, Herrlinger U, Jacobs A, Pechan PA, Rainov NG, Sena-Esteves M, Aghi M, Barnett FH, Chiocca EA, Breakefield XO: Therapeutic efficiency and safety of a second-generation replication-conditional HSVI vector for brain tumor gene therapy. Hum Gene Ther 1997, 8:2057-2068.

30. Bzik DJ, Fox BA, DeLuca NA, Person S: Nucleotide sequence specifying the glycoprotein gene, $g B$, of herpes simplex virus type I. Virology 1984, |33:30|-3|4.

31. Pellett PE, Kousoulas KG, Pereira L, Roizman B: Anatomy of the herpes simplex virus I strain $F$ glycoprotein B gene: primary sequence and predicted protein structure of the wild type and of monoclonal antibody-resistant mutants. J Virol 1985 , 53:243-253.

32. Bzik DJ, Fox BA, DeLuca NA, Person S: Nucleotide sequence of a region of the herpes simplex virus type I gB glycoprotein gene: mutations affecting rate of virus entry and cell fusion. Virology 1984, 137:185-190

33. Bond VC, Person S: Fine structure physical map locations of alterations that affect cell fusion in herpes simplex virus type I. Virology 1984, I32:368-376.

34. Debroy C, Pederson N, Person S: Nucleotide sequence of a herpes simplex virus type I gene that causes cell fusion. Virology 1985, 145:36-48.

35. Foster TP, Chouljenko VN, Kousoulas KG: Functional and Physical Interactions of the Herpes Simplex Virus Type-I (HSV-I) UL20 Membrane Protein with Glycoprotein K (gK). J Virol 2008 in press.

36. Foster TP, Melancon JM, Olivier TL, Kousoulas KG: Herpes simplex virus type I glycoprotein K and the UL20 protein are interdependent for intracellular trafficking and trans-Golgi network localization. J Virol 2004, 78: | 3262-13277.

37. Israyelyan AH, Melancon JM, Lomax LG, Sehgal I, Leuschner C, Kearney MT, Chouljenko VN, Baghian A, Kousoulas KG: Effective treatment of human breast tumor in a mouse xenograft model with herpes simplex virus type I specifying the NVI020 genomic deletion and the gBsyn 3 syncytial mutation enabling high viral replication and spread in breast cancer cells. Hum Gene Ther 2007, I 8:457-473.

38. Manservigi R, Spear PG, Buchan A: Cell fusion induced by herpes simplex virus is promoted and suppressed by different viral glycoproteins. Proc Natl Acad Sci USA 1977, 74:39|3-3917.

39. Tischer BK, von Einem J, Kaufer B, Osterrieder N: Two-step redmediated recombination for versatile high-efficiency markerless DNA manipulation in Escherichia coli. Biotechniques 2006, 40:19|-197.

40. Aslakson C], Miller FR: Selective events in the metastatic process defined by analysis of the sequential dissemination of subpopulations of a mouse mammary tumor. Cancer Res 1992 , 52:1399-I405.

4I. Kastrukoff LF, Lau AS, Puterman ML: Genetics of natural resistance to herpes simplex virus type I latent infection of the peripheral nervous system in mice. I Gen Virol 1986, 67(Pt 4):6|3-62|.

42. Lopez C: Genetics of natural resistance to herpesvirus infections in mice. Nature 1975, 258:152-153.

43. Shukla D, Dal Canto MC, Rowe CL, Spear PG: Striking similarity of murine nectin-Ialpha to human nectin-Ialpha $(\mathrm{HveC})$ in 
sequence and activity as a glycoprotein $D$ receptor for alphaherpesvirus entry. J Virol 2000, 74: I I773-I I78I.

44. Even DL, Henley AM, Geraghty RJ: The requirements for herpes simplex virus type I cell-cell spread via nectin-I parallel those for virus entry. Virus Res 2006, I I 9:195-207.

45. Reif AE: Some key problems for success of classical immunotherapy. In Immunity to Cancer Edited by: Reif AE, Mitchell EMS. New York: Academic Press; 1985:3-16.

46. Miller FR: Models of progression spanning preneoplasia and metastasis: the human MCFIOneoT.Tgn series and a panel of mouse mammary tumors subpopulations. In Mammary tumor cell cycle, Differentiation, and Metastasis Edited by: Dickson MELRB. Boston: Kluwer Academic; 1996:243-263.

47. Pulaski BA, Ostrand-Rosenberg S: Reduction of established spontaneous mammary carcinoma metastases following immunotherapy with major histocompatibility complex class II and B7.I cell-based tumor vaccines. Cancer Res 1998, 58:|486-|493.

48. Foster TP, Alvarez X, Kousoulas KG: Plasma membrane topology of syncytial domains of herpes simplex virus type I glycoprotein K (gK): the UL20 protein enables cell surface localization of gK but not gK-mediated cell-to-cell fusion. J Virol 2003, 77:499-5I0.

49. Foster TP, Rybachuk GV, Kousoulas KG: Glycoprotein K specified by herpes simplex virus type $I$ is expressed on virions as a Golgi complex-dependent glycosylated species and functions in virion entry. J Virol 200I, 75:1243I-12438.

Publish with Bio Med Central and every scientist can read your work free of charge

"BioMed Central will be the most significant development for disseminating the results of biomedical research in our lifetime. "

Sir Paul Nurse, Cancer Research UK

Your research papers will be:

- available free of charge to the entire biomedical community

- peer reviewed and published immediately upon acceptance

- cited in PubMed and archived on PubMed Central

- yours - you keep the copyright 